Research Article

\title{
A New Approach for Estimating Rock Discontinuity Trace Intensity Based on Rectangular Sampling Windows
}

\author{
Xiaoxue Huo $\mathbb{D}^{D}$, Qiong Wu $\mathbb{D}$, Huiming Tang, Zhen Meng $\mathbb{D}^{D}$, Di Wang $\mathbb{D}^{\text {, }}$, Yuxin Liu $\mathbb{D}^{\text {, }}$ \\ and Shiyu Li $(\mathbb{D}$
}

Faculty of Engineering, China University of Geosciences, Wuhan 430074, China

Correspondence should be addressed to Qiong Wu; wuqiong@cug.edu.cn

Received 22 September 2020; Revised 5 November 2020; Accepted 11 November 2020; Published 26 November 2020

Academic Editor: Chong Xu

Copyright $\odot 2020$ Xiaoxue Huo et al. This is an open access article distributed under the Creative Commons Attribution License, which permits unrestricted use, distribution, and reproduction in any medium, provided the original work is properly cited.

\begin{abstract}
Trace intensity is defined as mean total trace length of discontinuities per unit area, which is an important geometric parameter to describe fracture networks. The probability of each trace appearing in the sampling surface is different since discontinuity orientation has a scatter and is probabilistically distributed, so this factor should be taken into account in trace intensity estimation. This paper presents an approach to estimate the two-dimensional trace intensity by considering unequal appearing probability for discontinuities sampled by rectangular windows. The estimation method requires the number of discontinuities intersecting the window, the appearing probability of discontinuities with both ends observed, one end observed, and both ends censored, and the mean trace length of discontinuities intersecting the window. The new estimator is validated by using discontinuity data from an outcrop in Wenchuan area in China. Similarly, circular windows are used along with Mauldon's equation to calculate trace intensity using discontinuity trace data of the same outcrop as a contrast. Results indicate that the proposed new method based on rectangular windows shows close accuracy and less variability than that of the method based on circular windows due to the influence of finite sample size and the variability of location of the window and has advantage in application to sampling surfaces longer in one direction than in the other such as tunnel cross sections and curved sampling surfaces such as outcrops that show some curvature.
\end{abstract}

\section{Introduction}

The discontinuities have a significant influence on the stability, deformability, strength, and percolation characteristics of rock mass [1-5]. Characteristics of discontinuity and discontinuity sets are commonly inferred from discontinuity trace parameters such as trace intensity, length, and density. The aforementioned parameters can be used as typical geometric parameters to describe fracture networks by computer programs [6-9]. Trace intensity defined as mean total trace length of discontinuities per unit area, which is a pattern characteristic that incorporates both fracture density and mean length. Hence, intensity can substitute for dimensionally equivalent density as an input value of fracture networks programs. Intensity also can calculate equivalent mean spacing from a case of two-dimensional subparallel impersistent traces [10]. In addition, the estimation of intensity provides tests for determining whether fracture network is truly representative.
There are three types of intensities including linear, areal, and volumetric. The dimension of all types is $L^{-1}(L$ is length). The linear intensity is the simplest and most common type of intensity used for the whole discontinuities [11]. More recently, researches focus on volumetric intensity (discontinuity area per unit volume of rock mass). Zhang and Einstein [12] proposed an equation to calculate the mean fractures area per unit volume of the rock mass. Grossmann [13] proposed mathematical calculation method on volumetric intensity. Hekmatnejad et al. [14] addressed the problem of predicting the volumetric intensity in space and of quantifying the uncertainty in the true values, using information from observed discontinuities intersecting boreholes. Sanderson and Nixon [15] introduced some simple techniques to characterize the topology of a fracture network, which extended concepts of topological to 3 dimensions. No research has been done on estimation of trace intensity studied in this paper based on rectangular sampling windows. 
Several methods have been developed to estimate intensity on two-dimensional exposure. Zeeb et al. [16] reclassified the methods to acquire aforementioned geometric parameters into these: (1) scanline sampling (e.g., Priest and Hudson [17]), (2) window sampling (e.g., Paul [18]), and (3) circular estimator method (e.g., Mauldon et al. [19]). A few researchers have performed investigations on the method of estimating trace intensity in two-dimensional exposures. Ferrero and Umili [20] compared the existing fracture intensity estimation method and applied them to the North face of Aiguille du Marbree. Bandpey et al. [21] made a comparison of the methods for calculation geometrical characteristics of discontinuities in a cavern of the Rudbar Lorestan power plant. Kamali et al. [22] focused on fisher constant to research effect of shape and size of sampling window on the determination of intensity of trace discontinuity. Mahe et al. [23] applied mathematical calculations of fracture intensity on one site using measurements of three distinct fracture sets to evaluate and explain variability in collected data. Watkins et al. [24] developed a work flow for fracture data collection including fracture intensity in a region of heterogeneous fractures in a fold and thrust belt. Ortega et al.'s [25] used approach makes use of fracture-size distributions for two carbonate beds in Mexico and illustrates how size-cognizant measurements cast new light on widely accepted interpretation of geologic controls of fracture intensity. It can be seen from previous studies that a scanline survey allows a quick analysis of fracture network characteristics on outcrops, but it has size, truncation, and censoring biases [26]. The window sampling technique provides much data in a relatively short period of time and in the smallest part of the outcrop that is considered as one of the most important advantages of the window sampling technique.

This paper contains deduction of trace intensity estimators based on rectangular windows by considering that the probability of each trace appearing in the sampling surface is different. Based on previous studies on mean trace length estimation [27, 28], this paper focuses on the areal trace intensity estimation based on rectangular windows, which has advantage in application to sampling surfaces longer in one direction than in the other such as tunnel cross sections and curved sampling surfaces such as outcrops that show some curvature. The proposed new estimator is then validated by using discontinuity data from an outcrop in Wenchuan area in China. Similarly, circular windows are used along with Mauldon's equation to calculate trace intensity using discontinuity trace data of the same outcrop as a contrast.

\section{New Estimation Method of Trace Intensity Based on Rectangular Sampling Windows}

2.1. Deduction Process. Trace intensity is defined as mean total trace length of fractures per unit area [19]. According to the definition, trace intensity $(I)$ can be expressed as

$$
I=\frac{L^{\prime}}{w h}
$$

where $L^{\prime}$ is the total observed trace length of discontinuities in rectangular sampling window; $w$ and $h$ are, respectively, width and height of the rectangular window.

Depending on the position of the discontinuity, the intersection may occur in three ways: (a) both ends are censored, (b) one end is censored, and (c) both ends are observable in the window [28]. Let $N_{0}, N_{1}$, and $N_{2}$ denote the number of discontinuities with both ends censored, one end censored, and both ends observable, respectively. Let $L$ denote the total trace length of discontinuities intersecting the rectangular sampling window. Let $K$ denote the ratio of total observed trace length and total trace length of discontinuities intersecting the sampling window.

$$
K=\frac{L^{\prime}}{L} .
$$

In order to estimate $L^{\prime}$, we first deduct $K$ by considering these three types of discontinuities respectively:

2.1.1. Discontinuities with Both Ends Observable. Let $K_{1}$ denote the ratio of total observed trace length and total trace length of discontinuities intersecting the sampling window with both ends observable. It is easy to find that $K_{1}=1$.

2.1.2. Discontinuities with One End Censored. Let $K_{2}$ denote the ratio of total observed trace length and total trace length of discontinuities intersecting the sampling window with one end censored. Figure 1 shows a one end censored trace intersecting the lower boundary of the rectangular sampling window. Let $x_{i}$ denote the distance between the midpoint of the $i$ th trace and the lower boundary of the window. $x_{i}$ is positive when the midpoint is within the window, while $x_{i}$ is negative when the midpoint is out of the window. $\varphi_{i}$ denotes the apparent dip angle of the $i$ th trace. $l_{i}$ and $l_{i}^{\prime}$ are, respectively, the full length and observed length in the window of the $i$ th fracture trace. $K_{2}$ can be expressed as

$$
K_{2}=\frac{\sum_{i=1}^{N_{1}} l_{i}^{\prime}}{\sum_{i=1}^{N_{1}} l_{i}}=\frac{\sum_{i=1}^{N_{1}}\left(x_{i} / \sin \varphi_{i}\right)+\sum_{i=1}^{N_{1}}\left(l_{i} / 2\right)}{\sum_{i=1}^{N_{1}} l_{i}} .
$$

The apparent dip angle of discontinuities in the same set does not vary a lot, so (3) can be simplified as

$$
K_{2}=\frac{\sum_{i=1}^{N_{1}} l_{i}^{\prime}}{\sum_{i=1}^{N_{1}} l_{i}}=\frac{\left(\sum_{i=1}^{N_{1}} x_{i} / \sin \varphi_{i}\right)+\sum_{i=1}^{N_{1}}\left(l_{i} / 2\right)}{\sum_{i=1}^{N_{1}} l_{i}} .
$$

Assume that the midpoints of trace lengths are uniformly distributed in two-dimensional space; we can get the expression $\sum_{i=1}^{N_{1}} x_{i}=0$. So in this case, $K_{2}=1 / 2$. Similarly, we can obtain the same conclusion if these discontinuities with one end censored intersect the other boundaries of the sampling window.

2.1.3. Discontinuities with Both Ends Censored. Let $K_{3}$ denote the ratio of total observed trace length and total trace length of discontinuities intersecting the sampling window with both ends censored. Consider the problem of a 


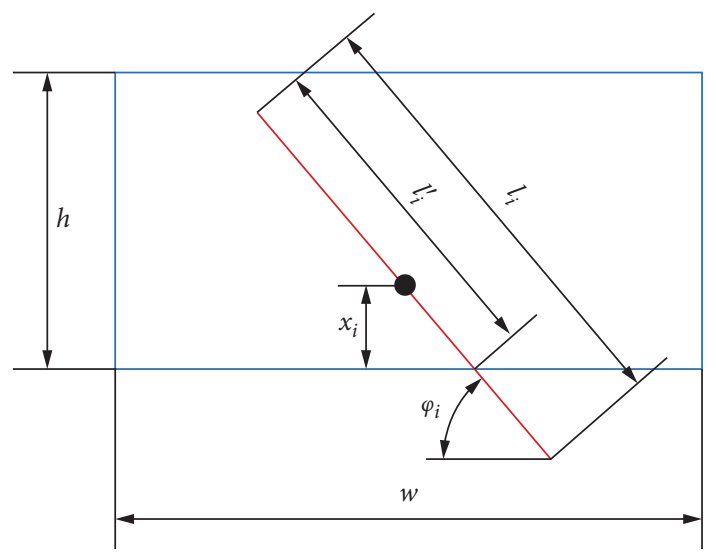

FIGURE 1: Intersection relationship between a trace with one end censored and the sampling window.

discontinuity with both ends censored with trace length, $l$, and apparent dip angle, $\varphi$. According to the intersection relationships between discontinuity and sampling window, four cases will be discussed in the following. They are

(1) $l \geq(h / \sin \varphi)$ and $\varphi \geq \tan ^{-1}(h / w)$ (Figure 2), (2) $l<(h / \sin \varphi)$ and $\varphi \geq \tan ^{-1}(h / w)$ (Figure 3), (3) $l \geq(h / \sin \varphi)$ and $\varphi<\tan ^{-1}(h / w)$ (Figure 4), and (4) $l<(h / \sin \varphi)$ and $\varphi<\tan ^{-1}(h / w)$ (Figure 5).

Let $K_{31}, K_{32}, K_{33}$, and $K_{34}$ denote the ratio of total observed trace length and total trace length of discontinuities in the aforementioned four cases, respectively. Use $P_{31}, P_{32}, P_{33}$, and $P_{34}$ as the probability of the traces appearing in the window, respectively, in each case. The equations of $P_{31}, P_{32}$, $P_{33}$, and $P_{34}$ are given as follows:

$$
\begin{aligned}
& P_{31}=\int_{\tan ^{-1}(h / w)}^{\infty} \int_{(h / \sin \varphi)}^{\infty} f(l, \varphi) \mathrm{d} l \mathrm{~d} \varphi, \\
& P_{32}=\int_{\tan ^{-1}(h / w)}^{\infty} \int_{0}^{(h / \sin \varphi)} f(l, \varphi) \mathrm{d} l \mathrm{~d} \varphi, \\
& P_{33}=\int_{0}^{\tan ^{-1}(h / w)} \int_{(h / \sin \varphi)}^{\infty} f(l, \varphi) \mathrm{d} l \mathrm{~d} \varphi, \\
& P_{34}=\int_{0}^{\tan ^{-1}(h / w)} \int_{0}^{(h / \sin \varphi)} f(l, \varphi) \mathrm{d} l \mathrm{~d} \varphi,
\end{aligned}
$$

where $f(l, \varphi)$ is the probability density function of $l$ and $\varphi$.

It is important to note that it is not necessary to have a befitting theoretical probability density function for the length and apparent dip angle of discontinuity to calculate $P_{31}, P_{32}, P_{33}$, and $P_{34}$ by $(5 \mathrm{a})-(5 \mathrm{~d})$. The summation sign can be used instead of the integral sign in the absence of a theoretical probability density function. Let $t$ be the total number of traces that satisfy the condition of each case. Then (5a)-(5d) can be replaced by

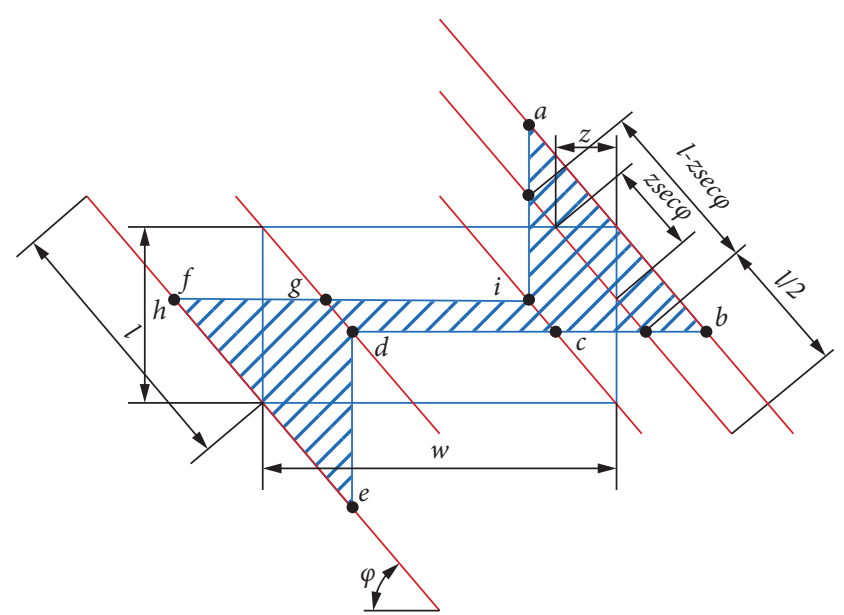

FIgURE 2: Discontinuities with both ends censored, $l \geq(h / \sin \varphi)$ and $\varphi \geq \tan ^{-1}(h / w)$.

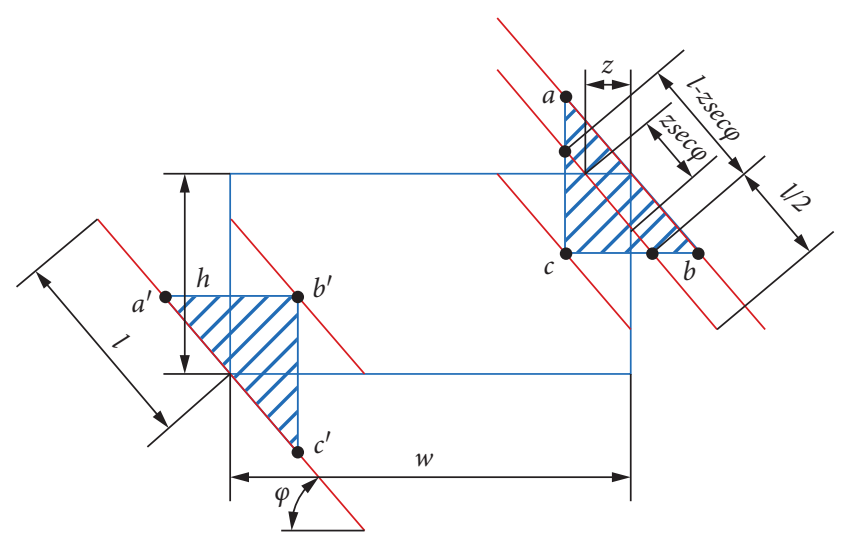

FIgURE 3: Discontinuities with both ends censored, $l<(h / \sin \varphi)$ and $\varphi \geq \tan ^{-1}(h / w)$.

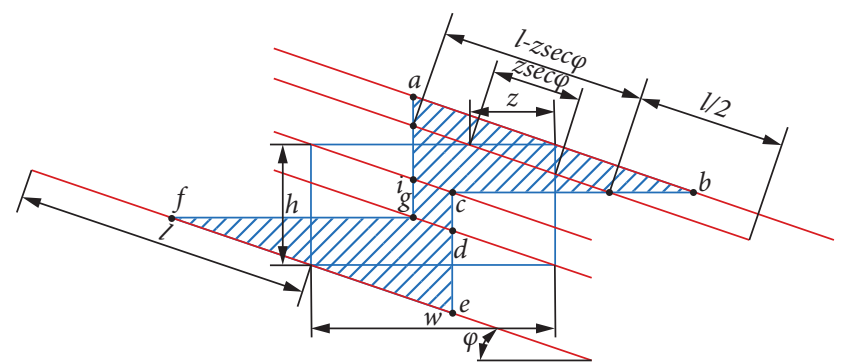

FIgURE 4: Traces with both ends censored, $l \geq(h / \sin \varphi)$ and $\varphi<\tan ^{-1}(h / w)$.

$$
\begin{aligned}
& P_{31} \approx \sum_{i=1}^{t} \operatorname{Rf}\left(l_{i}, \varphi_{i}\right), \\
& P_{32} \approx \sum_{i=1}^{t} \operatorname{Rf}\left(l_{i}, \varphi_{i}\right),
\end{aligned}
$$




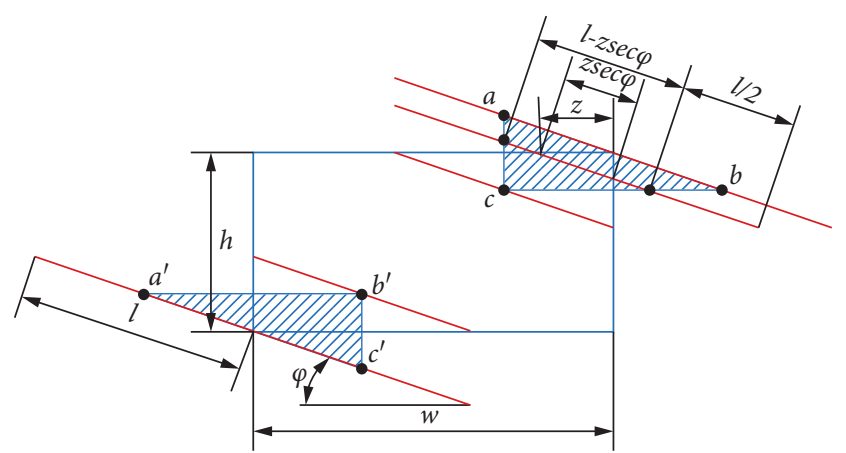

FIgURE 5: Traces with both ends censored, $l \geq(h / \sin \varphi)$ and $\varphi<\tan ^{-1}(h / w)$.

$$
\begin{aligned}
& P_{33} \approx \sum_{i=1}^{t} \operatorname{Rf}\left(l_{i}, \varphi_{i}\right), \\
& P_{34} \approx \sum_{i=1}^{t} \operatorname{Rf}\left(l_{i}, \varphi_{i}\right),
\end{aligned}
$$

where $\varphi_{i}$ denotes the apparent dip angle of the $i$ th trace in each case and $\operatorname{Rf}\left(l_{i}, \varphi_{i}\right)$ denotes the relative frequency of the $i$ th trace appearing on the window with length $l_{i}$ and the apparent dip angle $\varphi_{i} \cdot \operatorname{Rf}\left(l_{i}, \varphi_{i}\right)$ can be calculated as given below.

The probability of intersection between a discontinuity and a sampling domain is proportional to the volume $\left(V_{i}\right)$ within which the center of the discontinuity should lie in order to intersect the sampling domain [29]. According to the stated hypothesis, $\operatorname{Rf}\left(l_{i}, \varphi_{i}\right)$ can be calculated by (7)-(9) as given below.

$$
\begin{aligned}
V_{i}= & w h d_{i}\left[\cos ^{2} \theta_{i}+\sin ^{2} \theta_{i} \cos ^{2}\left(\alpha_{r}-\alpha_{i}\right)\right]^{0.5} \\
& +\frac{\pi d_{i}^{2}}{4}\left[w \sin \theta_{i}\left|\cos \left(\alpha_{r}-\alpha_{i}\right)\right|+h \cos \theta_{i}\right],
\end{aligned}
$$

where $\alpha_{i}$ and $\theta_{i}$ denote the dip direction and dip angle of the $i$ th discontinuity, respectively; $d_{i}$ is the diameter of the $i$ th discontinuity and $\alpha_{r}$ is the strike of the sampling window. In the absence of diameter values for discontinuities, a diameter value equal to $10 \%$ higher than the maximum trace length may be used for all the discontinuities in using (7).

The weighting function for the $i$ th discontinuity $\left(W_{i}\right)$ is given by

$$
W_{i}=\frac{1}{V_{i}}
$$

Then, the corrected relative frequency of the $i$ th discontinuity is given by

$$
\operatorname{Rf}\left(l_{i}, \varphi_{i}\right)=\frac{W_{i}}{\sum_{i=1}^{N} W_{i}}
$$

The derivations of $K_{31}, K_{32}, K_{33}$, and $K_{34}$ are given in the following:

(1) $l \geq(h / \sin \varphi)$ and $\varphi \geq \tan ^{-1}(h / w)$.

The shaded zone in Figure 2 shows the probable location of center point of traces in this case. In zone gicd, $\left(l_{i}^{\prime} / l_{i}\right)=(h / l \sin \varphi)$. And the area of zone gicd $S_{\text {gicd }}$ is

$$
S_{\text {gicd }}=(w-h \cot \varphi)(l \sin \varphi-h) .
$$

In the zone $a b c i,\left(l_{i}^{\prime} / l_{i}\right)=(z \sec \varphi / l)$. The meaning of $z$ is shown in Figure 2. And the area of zone abci $S_{a b c i}$ can be expressed as

$$
S_{a b c i}=\int_{0}^{h \cot \varphi}(l-z \sec \varphi) \sin \varphi \mathrm{d} z .
$$

On the whole shaded zone in Figure 2, the mean ratio (k31) of observed trace length and trace length of discontinuities with trace length $l$ and apparent dip angle $\varphi$ can be expressed as

$$
\begin{aligned}
k_{31} & =\frac{(h / l \sin \varphi)(w-h \cot \varphi)(l \sin \varphi-h)+2 \int_{0}^{h \cot \varphi}(z \sec \varphi / l)(l-z \sec \varphi) \sin \varphi \mathrm{d} z}{(w-h \cot \varphi)(l \sin \varphi-h)+2 \int_{0}^{h \cot \varphi}(l-z \sec \varphi) \sin \varphi \mathrm{d} z} \\
& =\frac{w h-\left(w h^{2} / l\right) \csc \varphi+\left(h^{3} / 3 l\right) \cot \varphi \csc \varphi}{w l \sin \varphi+h l \cos \varphi-w h} .
\end{aligned}
$$

For all the possible $l$ and $\varphi$ in this case, the ratio of total observed trace length and total trace length $K_{31}$ is

$$
K_{31}=\int_{\tan ^{-1}(h / w)}^{\infty} \int_{(h / \sin \varphi)}^{\infty} \frac{w h-\left(w h^{2} / l\right) \csc \varphi+\left(h^{3} / 3 l\right) \cot \varphi \csc \varphi}{w l \sin \varphi+h l \cos \varphi-w h} f(l, \varphi) \mathrm{d} l \mathrm{~d} \varphi .
$$


According to (6a)-(6d), probability density function in (13) can be estimated through replacing the integral sign by the summation sign using the empirical orientation distribution obtained for the discontinuity set. The simplified formula is as follows:

$$
K_{31} \approx \sum_{i=1}^{t} \frac{w h-\left(w h^{2} / l\right) \csc \varphi_{i}+\left(h^{3} / 3 l_{i}\right) \cot \varphi_{i} \csc \varphi_{i}}{w l_{i} \sin \varphi_{i}+h l_{i} \cos \varphi_{i}-w h} \operatorname{Rf}\left(l_{i}, \varphi_{i}\right)
$$

where $t, f(l, \varphi)$, and $\operatorname{Rf}\left(l_{i}, \varphi_{i}\right)$ have the same meaning as those in $(6 a)-(6 d)$.

(2) $l<(h / \sin \varphi)$ and $\varphi \geq \tan ^{-1}(h / w)$.

The shaded zone in Figure 3 shows the probable location of center point of traces in this case. The area of $a b c$ or $a^{\prime} b$ ' $c$ ' is

$$
S_{a b c}=S_{a^{\prime} b^{\prime} c^{\prime}}=\int_{0}^{l \cos \varphi}(l-z \sec \varphi) \sin \varphi \mathrm{d} z .
$$

In these shaded zones, $\left(l_{i}^{\prime} / l_{i}\right)=(z \sec \varphi / l)$, so the mean ratio $\left(k_{32}\right)$ of observed trace length and trace length of discontinuities with trace length $l$ and apparent dip angle $\varphi$ can be expressed as

$$
k_{32}=\frac{\int_{0}^{l \cos \varphi}(z \sec \varphi / l)(l-z \sec \varphi) \sin \varphi \mathrm{d} z}{\int_{0}^{l \cos \varphi}(l-z \sec \varphi) \sin \varphi \mathrm{d} z}=\frac{1}{3} .
$$

Therefore, for all the possible $l$ and $\varphi$ in this case, the ratio of total observed trace length and total trace length $K_{32}=1 / 3$.

(3) $l \geq(h / \sin \varphi)$ and $\varphi<\tan ^{-1}(h / w)$.

The shaded zone in Figure 4 shows the probable location of center point of traces in this case. In zone $i c d g,\left(l_{i}^{\prime} / l_{i}\right)=(w / l \cos \varphi)$. The area of zone $i c d g S_{i c d g}$ can be expressed as

$$
S_{i c d g}=(l \cos \varphi-w)(h-w \tan \varphi) .
$$

In zone $a b c i,\left(l_{i}^{\prime} / l_{i}\right)=(z \sec \varphi / l)$. Area abci $S_{a b c i}$ is

$$
S_{a b c i}=\int_{0}^{w}(l-z \sec \varphi) \sin \varphi \mathrm{d} z \text {. }
$$

So the mean ratio $\left(k_{33}\right)$ of observed trace length and trace length of discontinuities with trace length $l$ and apparent dip angle $\varphi$ can be expressed as

$$
\begin{aligned}
k_{33} & =\frac{(w / l \cos \varphi)(l \cos \varphi-w)(h-w \tan \varphi)+2 \int_{0}^{w}(z \sec \varphi / l)(l-z \sec \varphi) \sin \varphi \mathrm{d} z}{(l \cos \varphi-w)(h-w \tan \varphi)+2 \int_{0}^{w}(l-z \sec \varphi) \sin \varphi d z} \\
& =\frac{w h-\left(h w^{2} / l\right) \sec \varphi+\left(w^{3} / 3 l\right) \tan \varphi \sec \varphi}{h l \cos \varphi+w l \sin \varphi-w h} .
\end{aligned}
$$

For all the possible $l$ and $\varphi$ in this case, the ratio of total observed trace length and total trace length $K_{33}$ is

$$
K_{33}=\int_{0}^{\tan ^{-1}(h / w)} \int_{(h / \sin \varphi)}^{\infty} \frac{w h-\left(h w^{2} / l\right) \sec \varphi+\left(w^{3} / 3 l\right) \tan \varphi \sec \varphi}{h l \cos \varphi+w l \sin \varphi-w h} f(l, \varphi) \mathrm{d} l \mathrm{~d} \varphi .
$$

Similarly, a simplified formula of (20) can be expressed

as

$$
K_{33} \approx \sum_{i=1}^{t} \frac{w h-\left(h w^{2} / l_{i}\right) \sec \varphi_{i}+\left(w^{3} / 3 l_{i}\right) \tan \varphi_{i} \sec \varphi_{i}}{h l_{i} \cos \varphi_{i}+w l_{i} \sin \varphi_{i}-w h} \operatorname{Rf}\left(l_{i}, \varphi_{i}\right),
$$

where $t, f(l, \varphi)$, and $\operatorname{Rf}\left(l_{i}, \varphi_{i}\right)$ have the same meaning as those in $(6 a)-(6 d)$.

(4) $l \geq(h / \sin \varphi)$ and $\varphi<\tan ^{-1}(h / w)$.

The shaded zone in Figure 5 shows the probable location of center point of traces in this case. The area of zone $a b c$ or $a^{\prime} b^{\prime} c^{\prime}$ is

$$
S_{a b c}=S_{a^{\prime} b^{\prime} c^{\prime}}=\int_{0}^{l \cos \varphi}(l-z \sec \varphi) \sin \varphi \mathrm{d} z .
$$

In these shaded zones, $\left(l_{i}^{\prime} / l_{i}\right)=(z \sec \varphi / l)$, so the mean ratio $\left(k_{34}\right)$ of observed trace length and trace length of discontinuities with trace length $l$ and apparent dip angle $\varphi$ can be expressed as

$k_{34}=\frac{\int_{0}^{l \cos \varphi}(z \sec \varphi / l)(l-z \sec \varphi) \sin \varphi \mathrm{d} z}{\int_{0}^{l \cos \varphi}(l-z \sec \varphi) \sin \varphi \mathrm{d} z}=\frac{1}{3}$.

For all the possible $l$ and $\varphi$ in this case, the ratio of total observed trace length and total trace length $K_{34}=1 / 3$. 
By integrating the above four cases, $K_{3}$ can be obtained by the formula as follows:

$$
K_{3}=K_{31} P_{31}+K_{32} P_{32}+K_{33} P_{33}+K_{34} P_{34} .
$$

For all three types of discontinuities, the ratio of $\mathrm{L}^{\prime}$ and $L$ can be expressed as

$$
\frac{L^{\prime}}{L}=K=K_{1} P_{1}+K_{2} P_{2}+K_{3} P_{3}
$$

where

$$
\begin{aligned}
& P_{1}=\int_{\varphi_{i a}}^{\varphi_{s a}} \int_{l_{i a}}^{l_{s a}} f(l, \varphi) \mathrm{d} l \mathrm{~d} \varphi, \\
& P_{2}=\int_{\varphi_{i b}}^{\varphi_{s b}} \int_{l_{i b}}^{l_{s b}} f(l, \varphi) \mathrm{d} l \mathrm{~d} \varphi, \\
& P_{3}=\int_{\varphi_{i c}}^{\varphi_{s c}} \int_{l_{i c}}^{l_{s c}} f(l, \varphi) \mathrm{d} l \mathrm{~d} \varphi .
\end{aligned}
$$

In (25a)-(25d), $P_{1}, P_{2}$, and $P_{3}$ denote the probability of discontinuities of both ends observed (type a), one end observed (type b), and both ends censored (type c) intersecting the sampling window, respectively; $\varphi$ is apparent dip angle and $l$ is the length of discontinuities; $f(l, \varphi)$ is the probability density function of discontinuity orientation and length with $\varphi_{i} \leq \varphi \leq \varphi_{s}$ and $l_{i} \leq l \leq l_{s}$, where subscripts $i$ and $s$ denote inferior and superior limits, respectively; the subscripts $a, b, c$ indicate that the limits on $\varphi$ and $l$ are for the traces of type (a), type (b), and type (c), respectively.

From the abovementioned method, $P_{1}, P_{2}$, and $P_{3}$ can be simplified as follows:

$$
\begin{aligned}
& P_{1} \approx \sum_{i=1}^{N_{2}} \operatorname{Rf}\left(l_{i}, \varphi_{i}\right), \\
& P_{2} \approx \sum_{i=1}^{N_{1}} \operatorname{Rf}\left(l_{i}, \varphi_{i}\right), \\
& P_{3} \approx \sum_{i=1}^{N_{0}} \operatorname{Rf}\left(l_{i}, \varphi_{i}\right),
\end{aligned}
$$

where $N_{2}, N_{1}$, and $N_{0}$ denote the number of discontinuities of both ends observed (type a), one end observed (type b), and both ends censored (type c), respectively; $i$ denotes the $i$ th discontinuity in each type; $\operatorname{Rf}(l, \varphi)$ denotes the corrected relative frequency of discontinuities appearing on the window with length li and the apparent dip angle $\varphi_{i} . P_{1}, P_{2}$, and $P_{3}$ can be calculated by (7)-(9).

So intensify can be estimated by

$$
I=\frac{L^{\prime}}{w h}=\frac{\mu N K}{w h},
$$

where $\mu$ is the mean trace length of discontinuities; $w$ and $h$ are width and height of a rectangular window, respectively; $N$ denotes the total number of traces intersecting the window; $K$ can be estimated by (25a)-(25d).

The mean trace length of discontinuities $\mu$ can be calculated as given below [28]:

$$
\mu=\frac{w h\left(1+R_{0}-R_{2}\right)}{\left(1-R_{0}+R_{2}\right)(w B+h A)},
$$

where

$$
\begin{aligned}
& A=\int_{\alpha_{i}}^{\alpha_{s}} \int_{\theta_{i}}^{\theta_{s}}|\cos \varphi| f(\theta, \alpha) \mathrm{d} \theta \mathrm{d} \alpha \approx \sum_{i=1}^{N}\left[\left|\cos \varphi_{i}\right| \times \operatorname{Rf}\left(\varphi_{i}\right)\right], \\
& B=\int_{\alpha_{i}}^{\alpha_{s}} \int_{\theta_{i}}^{\theta_{s}}|\sin \varphi| f(\theta, \alpha) \mathrm{d} \theta \mathrm{d} \alpha \approx \sum_{i=1}^{N}\left[\left|\sin \varphi_{i}\right| \times \operatorname{Rf}\left(\varphi_{i}\right)\right],
\end{aligned}
$$

$$
\begin{aligned}
& \cos \varphi=\frac{1}{\left(1+\tan ^{2} \theta \cos ^{2} \delta\right)^{1 / 2}}, \\
& \sin \varphi=\frac{1}{\left(1+\cot ^{2} \theta \sec ^{2} \delta\right)^{1 / 2}} .
\end{aligned}
$$

In (28a)-(28e), $R_{0}$ and $R_{2}$ denote the fractions of discontinuities with both ends censored and both ends observable, respectively; $\theta$ and $\alpha$ are dip angle and dip direction; $f(\theta, \alpha)$ is the probability density function of discontinuity orientation with $\theta_{i} \leq \theta \leq \theta_{s}$ and $\alpha_{i} \leq \alpha \leq \alpha_{s}$, where subscripts $i$ and $s$ denote inferior and superior limits; $\delta$ denotes the acute angle between the dip direction and the vertical sampling plane; $\varphi_{i}$ denotes the apparent dip angle of the $i$ th trace; and $\operatorname{Rf}\left(\varphi_{i}\right)$ denotes the corrected relative frequency of the $i$ th trace appearing on the window with the apparent dip angle $\varphi_{i}$ resulting from a corresponding dip angle and a dip direction combination $(\theta, \alpha)$.

2.2. Simplification of $K_{3}$ in Practical Work. As deduced in Section 2.1, the estimation of $K_{3}$ needs the length of traces intersecting the window. However, it is hard to acquire the actual whole length for every trace in practical discontinuity sampling by windows with limited dimensions. Therefore, simplification on $K_{3}$ would be carried out to solve this problem as follows.

Consider a discontinuity with both ends censored of trace length $l \geq(h / \sin \varphi)$ and apparent $\operatorname{dip} \varphi \geq \tan ^{-1}(h / w)$ where the probable location of midpoint of trace is shown in Figure 6. Assume the ratio of observed trace length and trace length of discontinuities when the midpoint of the trace falls on the zone icm is the same as that on the zone abci (in the same way for the symmetric part of zone gnd and zone fgde). 
And assume that the mean ratio $\left(k_{31}\right)$ can be equal to average $1 / 3$ when the midpoint is inside the zone nimd. Thus (12) can be simplified as follows:

$$
k_{31} \approx \frac{(1 / 3)(w-l \cos \varphi)(l \sin \varphi-h)+2 \int_{0}^{l \cos \varphi}(z \sec \varphi / l)(l-z \sec \varphi) \sin \varphi \mathrm{d} z}{(w-l \cos \varphi)(l \sin \varphi-h)+2 \int_{0}^{l \cos \varphi}(l-z \sec \varphi) \sin \varphi \mathrm{d} z}=\frac{1}{3} .
$$

The simplification process of $k_{33}$ is the similar with $k_{31}$ as shown in Figure 7. Thus (19) can be simplified as given below:

$$
k_{33} \approx \frac{(1 / 3)(h-l \sin \varphi)(l \cos \varphi-w)+2 \int_{0}^{l \cos \varphi}(z \sec \varphi / l)(l-z \sec \varphi) \sin \varphi \mathrm{d} z}{(h-l \sin \varphi)(l \cos \varphi-w)+2 \int_{0}^{l \cos \varphi}(l-z \sec \varphi) \sin \varphi \mathrm{d} z}=\frac{1}{3}
$$

Thus, for all the possible $l$ and $\varphi$ in this case, the ratio of total observed trace length and total trace length $K_{31}=K_{33}=1 / 3$. So $K_{3}=1 / 3$.

Then,

$$
I=\frac{\mu N\left(P_{1}+(1 / 2) P_{2}+(1 / 3) P_{3}\right)}{w h},
$$

where $\mu, N, w$, and $h$ have the same meaning as those in (25a)-(25d); $P_{1}, P_{2}$, and $P_{3}$ can be calculated by (25a)-(25d); $\mu$ can be calculated by (28a)-(28e).

The simplification is based on the fact that the number of discontinuities with both ends censored (type c) in the window is relatively small compared to the other two types. If this condition is contrary to the fact, it will be indicated that the window dimensions used for surveying may be too small. The unsuitability of measurement range may influence the evaluation of the trace intensity of discontinuities, and the sampling bias would be still unquenchable even if complicated formulas are adopted. In addition, the midpoints of the trace have slim chance of falling in the simplified region. In conclusion, the simplification of $K_{3}$ in this section is reasonable.

\section{Case Study}

3.1. Application of the Proposed New Method Based on Rectangular Windows. The Wenchuan earthquake triggered enormous landslides in China, 2008. An almost vertical rock slope caused by the earthquake is an excellent exposed outcrop to investigate the discontinuity geometry characteristics of the rock mass, which is significant to better understand the formation mechanism of earthquake landslides. The discontinuity trace network of the outcrop as shown in Figure 8 was used to estimate rock discontinuity mean trace length and density in a previous study [27]. The discontinuity data of this outcrop are utilized in this section to validate the proposed new method based on rectangular windows. According to the orientation data, discontinuities on the outcrop can be divided into two sets. Rectangular sampling windows with dimensions of $90 \mathrm{~m} \times 45 \mathrm{~m}$, $110 \mathrm{~m} \times 55 \mathrm{~m}$, and $130 \mathrm{~m} \times 65 \mathrm{~m}$ have been placed at four locations on the outcrop. Trace intensity values, respectively, for two sets of discontinuities sampled by all twelve windows are estimated according to the methodology (in (31)) explained in Section 2. The estimation results are presented in Table 1 and Figure 9. The obtained overall mean value and coefficient of variation related to set 1 are equal to $0.151 \mathrm{~m}^{-1}$ and 0.299 and the ones related to set 2 are equal to $0.102 \mathrm{~m}^{-1}$ and 0.205 , respectively. True intensity values are calculated by using the data of trace length for discontinuities intersecting sampling windows on the outcrop. The obtained true mean values for all the windows related to set 1 and set 2 are also shown in Table 1 and Figure 9. Both the estimated and the true values indicate that the discontinuity trace intensity varies with the size and the location of the rectangular windows.

An estimation error can be defined as

$$
\operatorname{error}_{I}=\frac{I_{t}-I_{e}}{I_{t}} \times 100 \% \text {, }
$$

where $I_{t}$ and $I_{e}$ denote the true and estimated trace intensity values, respectively. This error is to evaluate the estimation accuracy of the proposed method. In Table 1 and Figure 10, the estimation errors for set 1 and set 2 related to different sizes of rectangular windows at different locations are presented. The error range caused by finite sample sizes and spatial variability for rectangular window is $-36.3 \%$ to $+14.0 \%$ for set 1 and $-38.9 \%$ to $+9.6 \%$ for set 2 .

\subsection{Application of the Method Based on Circular Windows.}

The equations (in (33)) to estimate trace intensity using circular windows proposed by Mauldon [6] are also used to estimate the trace intensity for discontinuities on the same outcrop. 


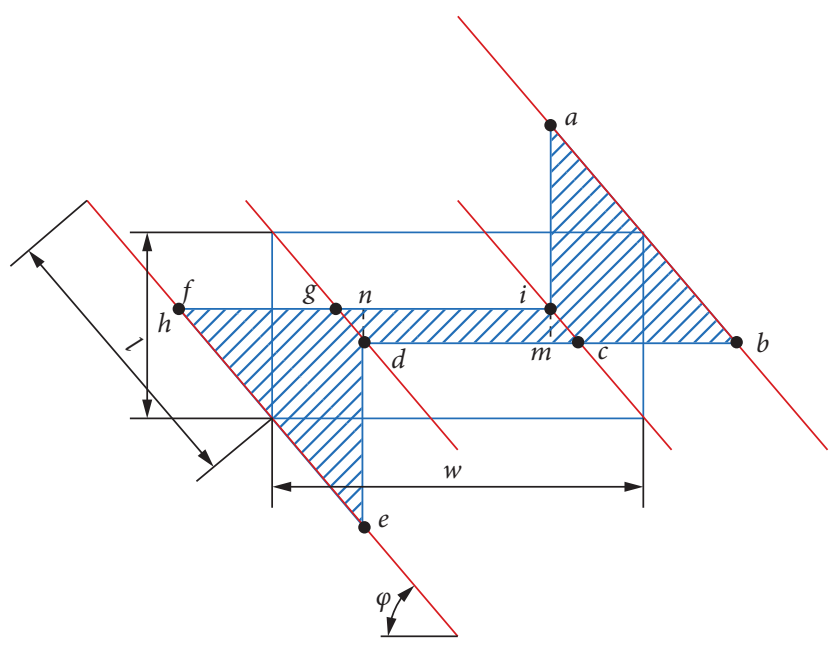

Figure 6: Discontinuities with both ends censored, $l \geq(h / \sin \varphi)$ and $\varphi \geq \tan ^{-1}(h / w)$.

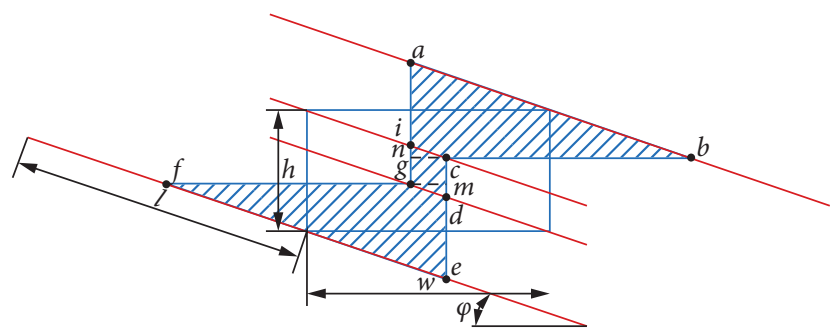

FIGURE 7: Traces with both ends censored, $l \geq(h / \sin \varphi)$ and $\varphi \geq \tan ^{-1}(h / w)$.

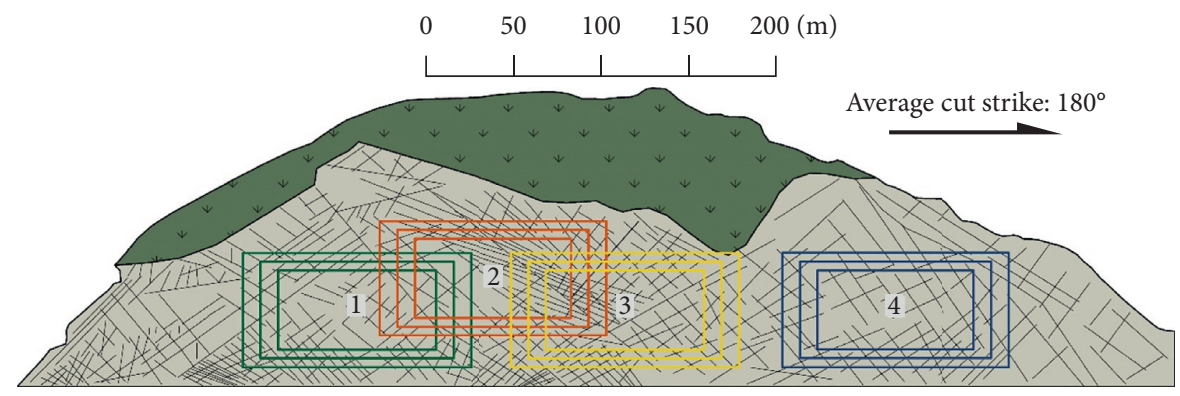

Figure 8: Sizes and locations of rectangular windows used to estimate mean trace length [27].

$$
I=\frac{N-N_{C}+N_{T}}{4 R}
$$

where $N$ denotes the total number of traces intersecting the circular window, $N_{C}$ denotes the number of traces that are contained in the circular window, and $N_{T}$ denotes the number of traces that transect the circular window.

A series of circular windows with radii of $20 \mathrm{~m}, 30 \mathrm{~m}$, and $40 \mathrm{~m}$ at five locations were placed on the discontinuity trace network as shown in Figure 11. The calculation results of trace intensity according to (33) are shown in Table 2 and Figure 12. The overall mean value and coefficient of variation related to set 1 are equal to $0.139 \mathrm{~m}^{-1}$ and 0.482 and the ones related to set 2 are equal to $0.095 \mathrm{~m}^{-1}$ and 0.324 , respectively. The obtained true values are for all the circular windows related to set 1 and set 2 are also shown in Table 2 and
Figure 12. Both the estimated and the true values indicate that the discontinuity trace intensity also varies with the size and the location of the circular windows. The calculating methods of estimation errors were the same as that illustrated in Section 3.1. Table 2 and Figure 13 depict the estimation errors of Mauldon's method using circular windows. The error range for circular windows is $-56.1 \%$ to $+52.0 \%$ for set 1 and $-57.2 \%$ to $+38.4 \%$ for set 2 .

3.3. Comparison of Results from the Two Intensity Estimation Methods. Errors of overall mean predictions obtained through the rectangular windows method were close to that obtained through the circular windows method. The error ranges obtained for Mauldon's method based on the circular windows are larger than that resulting from the proposed method based 
TABLE 1: Estimated intensity and its \% error based on rectangular sampling windows.

\begin{tabular}{|c|c|c|c|c|c|c|}
\hline \multirow[t]{2}{*}{ Window size $(\mathrm{m}) /$ location number } & \multicolumn{2}{|c|}{$\begin{array}{c}\text { Estimated value } \\
\left(m^{-1}\right)\end{array}$} & \multicolumn{2}{|c|}{$\begin{array}{l}\text { True value } \\
\left(\mathrm{m}^{-1}\right)\end{array}$} & \multicolumn{2}{|c|}{$\%$ error of $I_{\mathrm{e}}$} \\
\hline & Set 1 & Set 2 & Set 1 & Set 2 & Set 1 & Set 2 \\
\hline $90 \times 45 /$ location 1 & 0.114 & 0.092 & 0.084 & 0.088 & -36.3 & -4.8 \\
\hline $90 \times 45 /$ location 2 & 0.223 & 0.073 & 0.227 & 0.053 & 1.6 & -38.9 \\
\hline $90 \times 45 /$ location 3 & 0.182 & 0.152 & 0.189 & 0.125 & 3.5 & -21.8 \\
\hline $90 \times 45 /$ location 4 & 0.093 & 0.111 & 0.081 & 0.082 & -14.9 & -34.9 \\
\hline $110 \times 55 /$ location 1 & 0.128 & 0.096 & 0.096 & 0.091 & -33.8 & -6.1 \\
\hline $110 \times 55 /$ location 2 & 0.207 & 0.091 & 0.216 & 0.069 & 4.3 & -32.4 \\
\hline $110 \times 55 /$ location 3 & 0.179 & 0.128 & 0.179 & 0.126 & 0.4 & -1.7 \\
\hline $110 \times 55 /$ location 4 & 0.101 & 0.091 & 0.084 & 0.085 & -20.0 & -7.1 \\
\hline $130 \times 65 /$ location 1 & 0.142 & 0.097 & 0.106 & 0.093 & -34.7 & -3.7 \\
\hline $130 \times 65 /$ location 2 & 0.174 & 0.088 & 0.203 & 0.078 & 14.0 & -13.2 \\
\hline $130 \times 65 /$ location 3 & 0.171 & 0.110 & 0.173 & 0.122 & 1.2 & 9.6 \\
\hline $130 \times 65 /$ location 4 & 0.092 & 0.096 & 0.086 & 0.087 & -6.9 & -10.0 \\
\hline Mean value & 0.151 & 0.102 & 0.144 & 0.091 & 4.8 & 11.6 \\
\hline Coefficient of variation & 0.299 & 0.205 & 0.409 & 0.247 & & \\
\hline Range & & & & & -36.3 to 14.0 & -38.9 to 9.6 \\
\hline
\end{tabular}

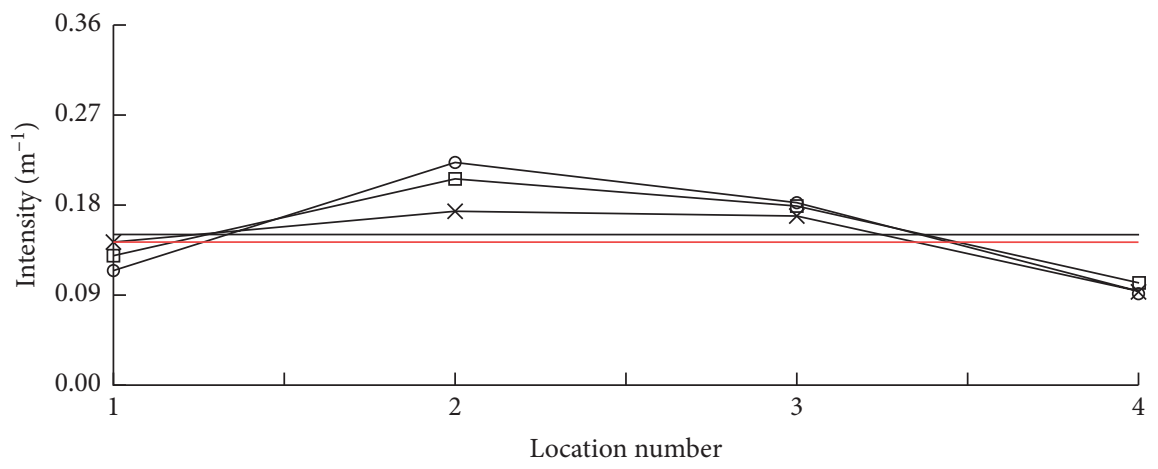
- $90 \times 45$
_ Overall estimated mean
- $100 \times 55$
_ Overall true mean

$\times 130 \times 65$

(a)

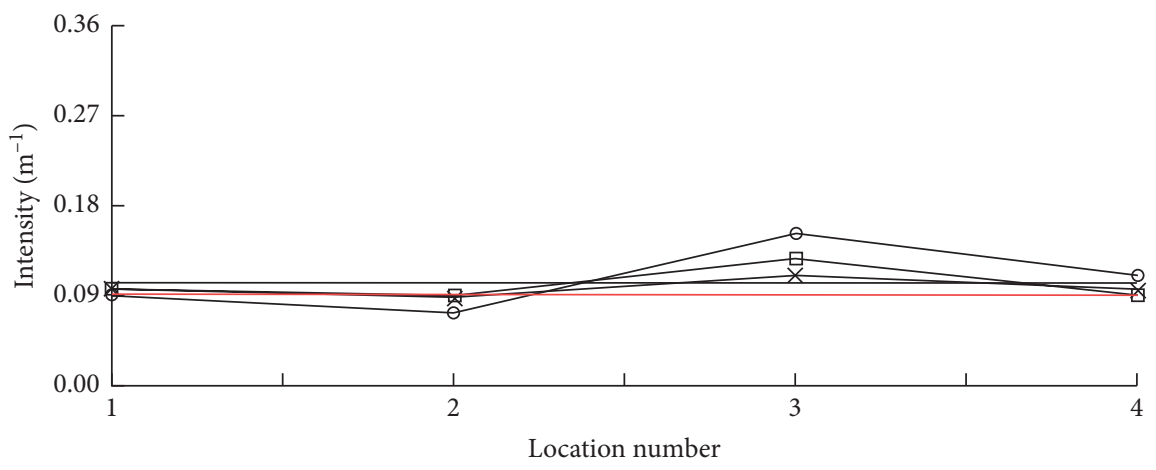
๑ $90 \times 45$
_ Overall estimated mean
$\square-100 \times 55$
_ Overall true mean

$\leftarrow 130 \times 65$

(b)

FIGURE 9: Estimated intensity based on new estimation method using rectangular windows of different sizes placed at different locations on the outcrop. (a) Set 1. (b) Set 2. 


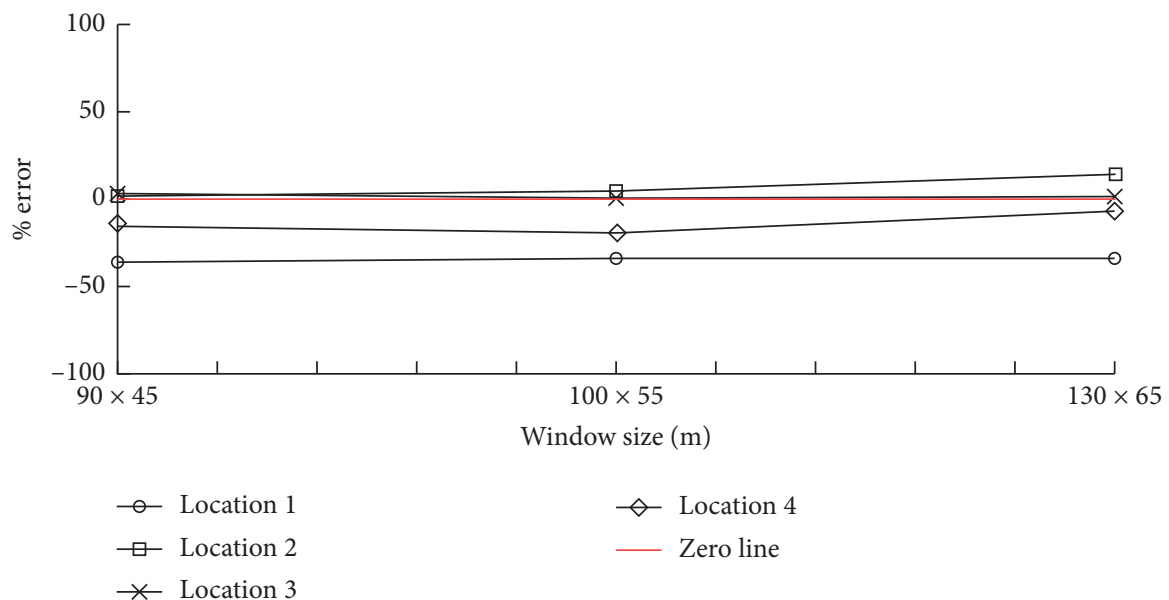

(a)

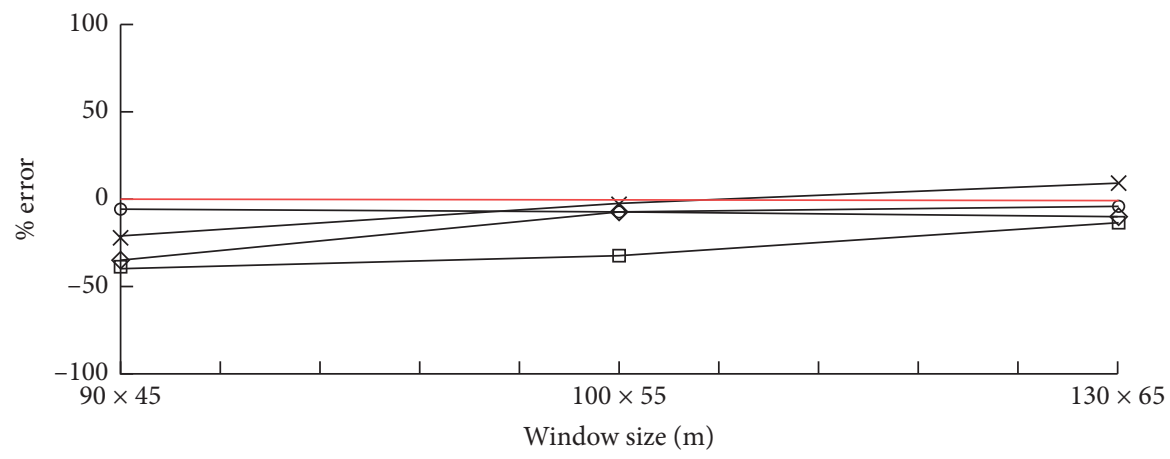

$\begin{array}{ll}- \text { Location } 1 & \diamond \text { Location } 4 \\ \square \text { Location } 2 & - \text { Zero line } \\ \leftarrow \text { Location } 3 & \end{array}$

(b)

Figure 10: Prediction error of mean trace length associated with finite sample sizes and spatial variability based on new estimation method using rectangular windows of different sizes placed at different locations on the outcrop. (a) Set 1. (b) Set 2.

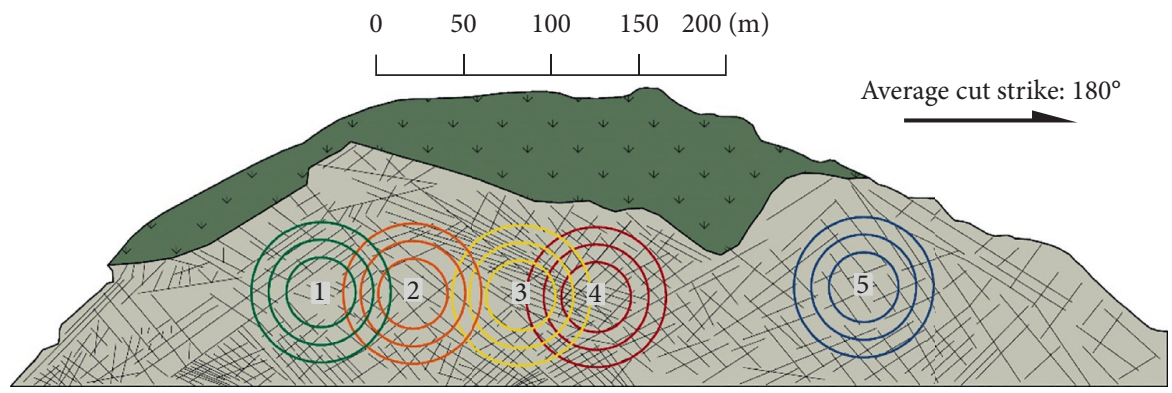

FIgURE 11: Sizes and locations of the circular windows used to estimate mean trace length [27].

TABLE 2: Estimated intensity and its \% error based on circular sampling windows.

\begin{tabular}{|c|c|c|c|c|c|c|}
\hline \multirow[t]{2}{*}{ Window size $(m) /$ location number } & \multicolumn{2}{|c|}{$\begin{array}{c}\text { Estimated } \\
\text { value }\left(m^{-1}\right)\end{array}$} & \multicolumn{2}{|c|}{ True value $\left(\mathrm{m}^{-1}\right)$} & \multicolumn{2}{|c|}{$\%$ error of $I_{\mathrm{e}}$} \\
\hline & Set 1 & Set 2 & Set 1 & Set 2 & Set 1 & Set 2 \\
\hline 20/location 1 & 0.063 & 0.125 & 0.071 & 0.089 & -31.7 & -31.5 \\
\hline 20/location 2 & 0.088 & 0.100 & 0.114 & 0.070 & 11.9 & -0.9 \\
\hline
\end{tabular}


TABle 2: Continued.

\begin{tabular}{|c|c|c|c|c|c|c|}
\hline \multirow[t]{2}{*}{ Window size $(m) /$ location number } & \multicolumn{2}{|c|}{$\begin{array}{c}\text { Estimated } \\
\text { value }\left(m^{-1}\right)\end{array}$} & \multicolumn{2}{|c|}{ True value $\left(\mathrm{m}^{-1}\right)$} & \multicolumn{2}{|c|}{$\%$ error of $I_{\mathrm{e}}$} \\
\hline & Set 1 & Set 2 & Set 1 & Set 2 & Set 1 & Set 2 \\
\hline 20/location 3 & 0.275 & 0.100 & 0.281 & 0.069 & 6.7 & -57.2 \\
\hline 20/location 4 & 0.213 & 0.150 & 0.254 & 0.143 & -43.1 & 5.3 \\
\hline 20/location 5 & 0.088 & 0.038 & 0.092 & 0.057 & 52.0 & 8.3 \\
\hline 30/location 1 & 0.075 & 0.125 & 0.063 & 0.109 & -56.1 & 9.3 \\
\hline $30 /$ location 2 & 0.075 & 0.108 & 0.087 & 0.079 & -12.5 & 17.5 \\
\hline 30/location 3 & 0.233 & 0.067 & 0.261 & 0.075 & -1.6 & -35.8 \\
\hline 30/location 4 & 0.183 & 0.108 & 0.207 & 0.123 & -28.5 & 4.2 \\
\hline 30/location 5 & 0.075 & 0.042 & 0.078 & 0.050 & -31.4 & -30.0 \\
\hline 40/location 1 & 0.119 & 0.106 & 0.083 & 0.110 & -25.3 & -4.2 \\
\hline 40/location 2 & 0.131 & 0.063 & 0.109 & 0.083 & -13.6 & 38.4 \\
\hline 40/location 3 & 0.206 & 0.100 & 0.232 & 0.081 & -20.0 & -13.8 \\
\hline 40/location 4 & 0.181 & 0.113 & 0.187 & 0.121 & -2.8 & 5.1 \\
\hline 40/location 5 & 0.088 & 0.075 & 0.081 & 0.061 & 6.1 & -14.3 \\
\hline Mean value & 0.139 & 0.095 & 0.147 & 0.088 & -4.9 & 7.4 \\
\hline Coefficient of variation & 0.482 & 0.324 & 0.529 & 0.299 & & \\
\hline Range & & & & & -56.1 to 52.0 & -57.2 to 38.4 \\
\hline
\end{tabular}

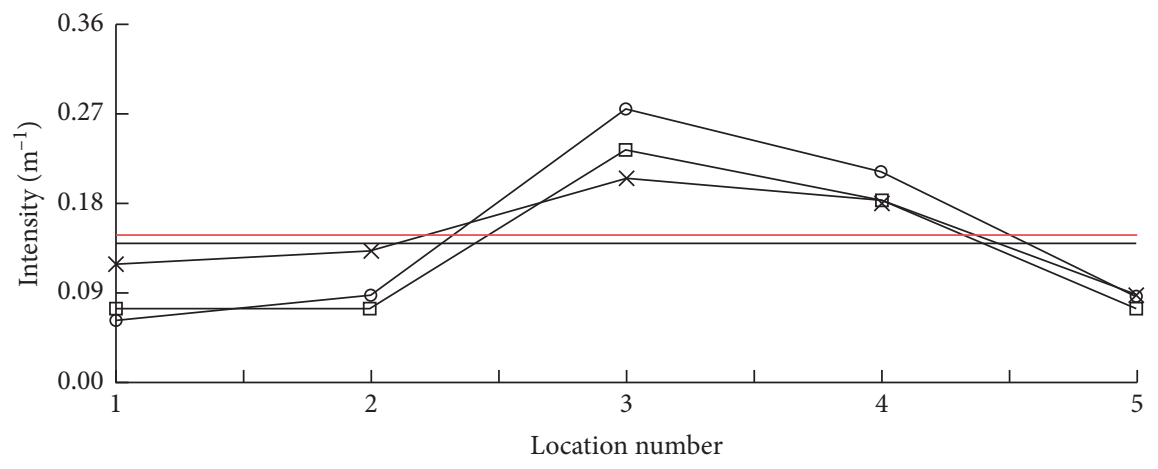
- $R=20$
_ Overall estimated mean
$\square R=30$
_ Overall true mean
$\times R=40$

(a)

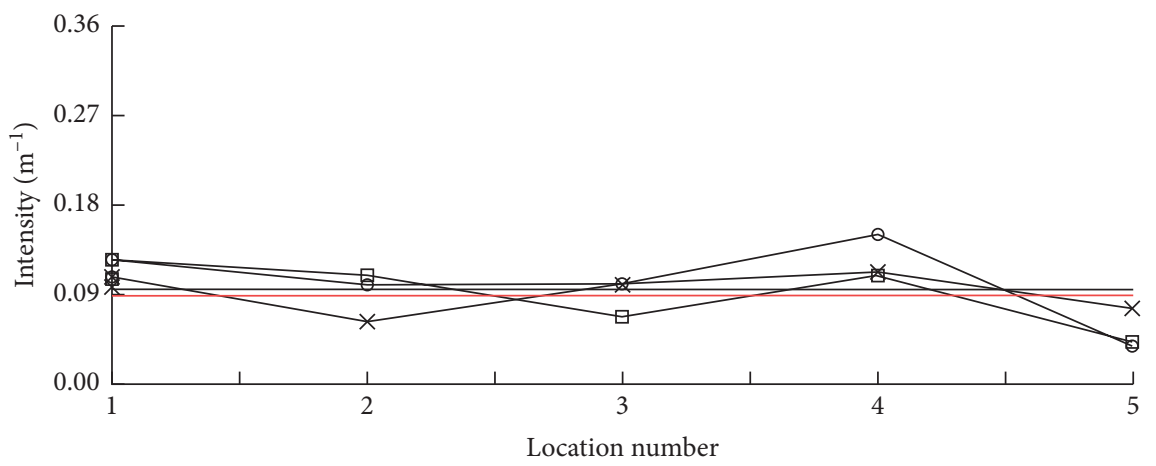
- $-R=20$
- Overall estimated mean
$\square R=30$
_ Overall true mean
$\star R=40$

(b)

Figure 12: Estimated intensity based on Mauldon's equation using circular windows of different sizes placed at different locations on the outcrop. (a) Set 1. (b) Set 2. 


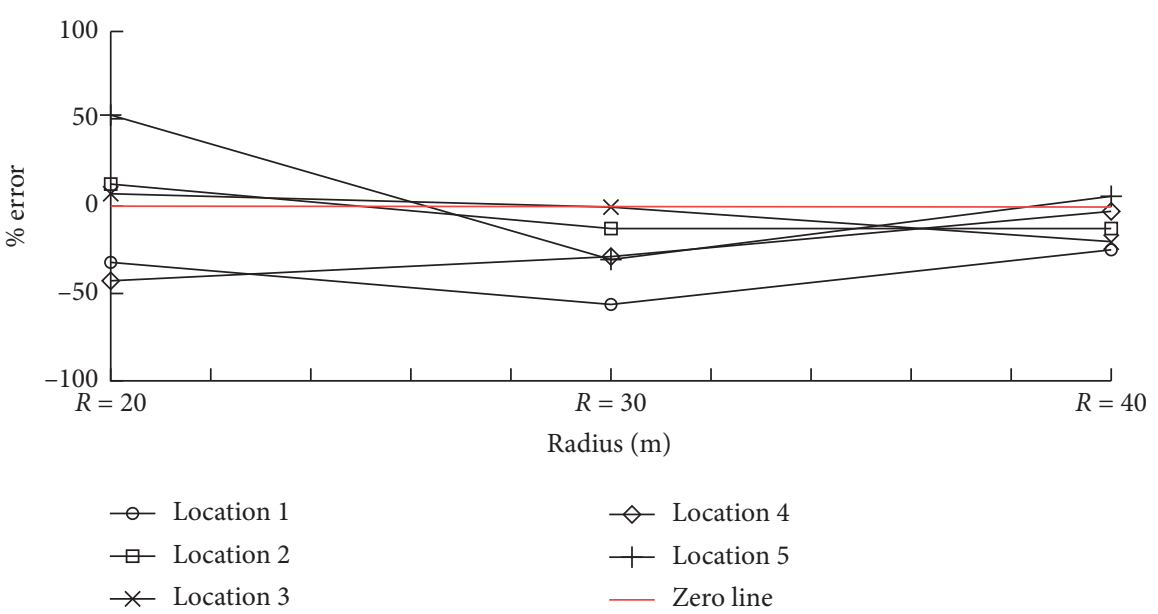

(a)

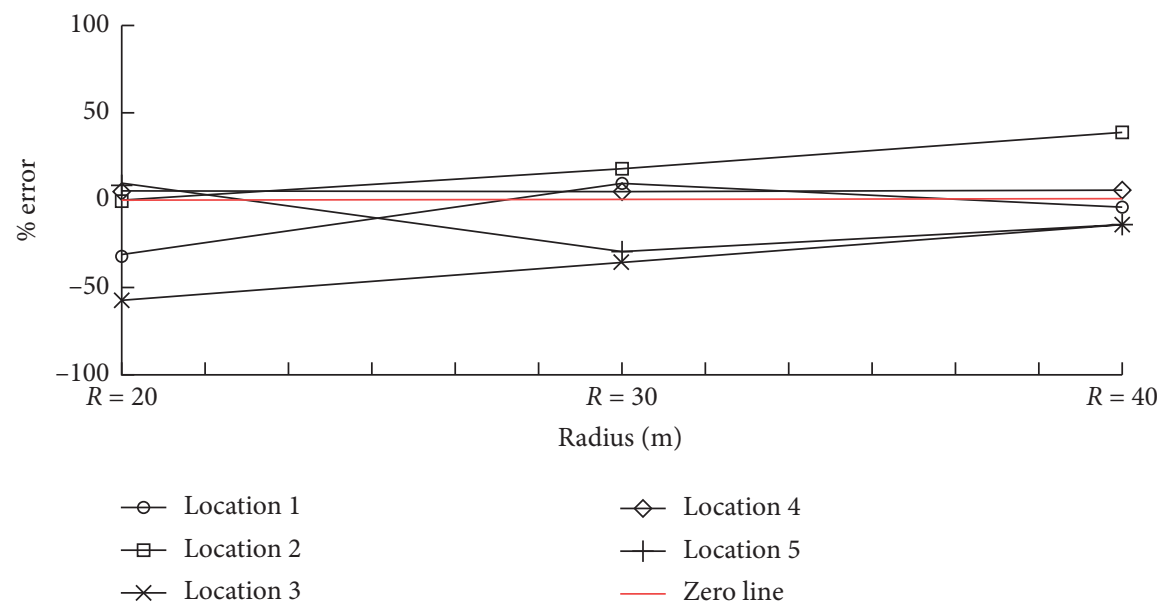

(b)

Figure 13: Prediction error of intensity associated with finite sample sizes and spatial variability based on Mauldon's equation using circular windows of different sizes placed at different locations on the outcrop. (a) Set 1. (b) Set 2.

on rectangular windows. The coefficient of variation values depicted in Tables 1 and 2 also indicates that the estimation results based on the proposed method show less variability than that of the method based on circular windows due to the variability of window location and size. The proposed method allows one to incorporate the relative frequency of each trace appearing on the exposure. Because this equation uses the angle between the discontinuity plane and the exposure plane, it can also be utilized to estimate trace intensity for traces on curved sampling surfaces such as outcrops that show some curvature. In this case, the curved surface could be divided into several planar surfaces. Then the relative frequencies can be estimated for the traces appearing on each planar surface. Besides, rectangular window has advantage in sampling discontinuities on rock mass surfaces longer in one direction than in the other such as tunnel cross sections.

\section{Conclusions}

Trace intensity is one of the most important characteristics of fractures that affect the mechanical properties of the rock mass.
In this study, a new estimation method of discontinuity trace intensity based on rectangular windows has been proposed by considering that the probability of each trace appearing on the two-dimensional exposures is different. The discontinuity traces on the outcrop in Wenchuan area were used along with rectangular windows to estimate the trace intensity using the proposed equations. Similarly, circular windows were used along with Mauldon's equation [19] to calculate the trace intensity for the discontinuity traces appearing in the same outcrop as a comparison. Errors of overall mean predictions obtained through the rectangular window method were close to that obtained through the circular window method. The error ranges obtained for Mauldon's method based on the circular windows are larger than that resulting from the proposed method based on rectangular windows. The coefficient of variation values depicted in Tables 1 and 2 also indicates that the estimation results based on the proposed method show less variability than that of the method based on circular window due to the variability of window location and size. It should be note that the proposed method based on rectangular sampling windows has advantage in application to 
sampling surfaces longer in one direction than in the other such as tunnel cross sections and curved sampling surfaces such as outcrops that show some curvature.

\section{Data Availability}

The orientation data used to support the findings of this study are available from the corresponding author upon request.

\section{Conflicts of Interest}

The authors declare that they have no conflicts of interest.

\section{Acknowledgments}

This research was funded by the National Key R\&D Program of China (nos. 2019YFC1509705 and 2017YFC1501301), National Natural Science Foundation of China (no. 41877259) and the Natural Science Foundation of Hubei Province of China (no. 2018CFB666). The authors are grateful to these organizations for providing financial support.

\section{References}

[1] International Society for Rock Mechanics Commission on Standardization of Laboratory and Field Tests, "Suggested methods for the quantitative description of discontinuities in rock masses," International Journal of Rock Mechanics and Mining Sciences \& Geomechanics Abstracts, vol. 15, no. 6, pp. 319-368, 1978.

[2] Q. Wu, Y. Xu, H. Tang et al., "Peak shear strength prediction for discontinuities between two different rock types using a neural network approach," Bulletin of Engineering Geology and the Environment, vol. 78, no. 4, pp. 2315-2329, 2018.

[3] Q. Wu, Y. Xu, H. Tang et al., "Investigation on the shear properties of discontinuities at the interface between different rock types in the Badong formation, China," Engineering Geology, vol. 245, pp. 280-291, 2018.

[4] Q. Wu, Y. Jiang, H. Tang et al., "Experimental and numerical studies on the evolution of shear behaviour and damage of natural discontinuities at the interface between different rock types," Rock Mechanics and Rock Engineering, vol. 53, no. 8, pp. 3721-3744, 2020.

[5] H.-m. Tang, J. Wasowski, and C. H. Juangc, "Geohazards in the three gorges reservoir area, China-lessons learned from decades of research," Engineering Geology, vol. 261, Article ID 105267, 2019.

[6] J. Zheng, Y. Zhao, Q. Lü, T. Liu, J. Deng, and R. Chen, "Estimation of the three-dimensional density of discontinuity systems based on one-dimensional measurements," International Journal of Rock Mechanics and Mining Sciences, vol. 94, pp. 1-9, 2017.

[7] J. Zheng, X. Wang, Q. Lü et al., "A contribution to relationship between volumetric joint count (jv) and rock quality designation (RQD) in three-dimensional (3-D) space," Rock Mechanics and Rock Engineering, vol. 53, no. 3, pp. 1485-1494, 2020.

[8] M. Mauldon, "Intersection probabilities of impersistent joints," International Journal of Rock Mechanics and Mining Sciences \& Geomechanics Abstracts, vol. 31, no. 2, pp. 107-115, 1994.
[9] A. Becker and M. R. Gross, "Mechanism for joint saturation in mechanically layered rocks: an example from southern Israel," Tectonophysics, vol. 257, no. 2-4, pp. 223-237, 1996.

[10] W. S. Dershowitz and H. H. Herda, "Interpretation of fracture spacing and intensity," in Proceedings of the 33rd U.S. Symposium on Rock Mechanics, pp. 757-766, Rotterdam, Netherlands, January 1992.

[11] M. B. Rohrbaugh Jr., W. M. Dunne, and M. Mauldon, "Estimating fracture trace intensity, density, and mean length using circular scan lines and windows," AAPG Bulletin, vol. 86, no. 12, pp. 2089-2104, 2002.

[12] L. Zhang and H. H. Einstein, "Estimating the intensity of rock discontinuities," International Journal of Rock Mechanics and Mining Sciences, vol. 37, no. 5, pp. 819-837, 2000.

[13] N. F. Grossmann, "About the determination of the joint set intensity," in Proceedings of Harmonising Rock Engineering and the Environment, pp. 421-422, Beijing, China, October 2011.

[14] A. Hekmatnejad, X. Emery, A. Brzovic, P. Schachter, and J. A. Vallejos, "Spatial modeling of discontinuity intensity from borehole observations at El Teniente mine, Chile," Engineering Geology, vol. 228, pp. 97-106, 2017.

[15] D. J. Sanderson and C. W. Nixon, "The use of topology in fracture network characterization," Journal of Structural Geology, vol. 72, pp. 55-66, 2015.

[16] C. Zeeb, E. Gomez-Rivas, P. D. Bons, S. Virgo, and P. Blum, "Fracture network evaluation program (FraNEP): a software for analyzing 2D fracture trace-line maps," Computers \& Geosciences, vol. 60, pp. 11-22, 2013.

[17] S. D. Priest and J. A. Hudson, "Estimation of discontinuity spacing and trace length using scanline surveys," International Journal of Rock Mechanics and Mining Sciences \& Geomechanics Abstracts, vol. 18, no. 3, pp. 183-197, 1981.

[18] P. J. Pahl, "Estimating the mean length of discontinuity traces," International Journal of Rock Mechanics and Mining Sciences \& Geomechanics Abstracts, vol. 18, no. 3, pp. 221-228, 1981.

[19] M. Mauldon, W. M. Dunne, and M. B. Rohrbaugh Jr., "Circular scanlines and circular windows: new tools for characterizing the geometry of fracture traces," Journal of Structural Geology, vol. 23, no. 2-3, pp. 247-258, 2001.

[20] A. M. Ferrero and G. Umili, "Comparison of methods for estimating fracture size and intensity applied to Aiguille Marbrée (Mont Blanc)," International Journal of Rock Mechanics and Mining Sciences, vol. 48, no. 8, pp. 1262-1270, 2011.

[21] A. K. Bandpey, K. Shahriar, M. Sharifzadeh, and P. Marefvand, "Comparison of methods for calculating geometrical characteristics of discontinuities in a cavern of the Rudbar Lorestan power plant," Bulletin of Engineering Geology and the Environment, vol. 78, no. 2, pp. 1073-1093, 2019.

[22] A. Kamali, K. Shahriar, M. Sharifzadeh, A. Aalianvari, and A. Esmaeilzadeh, "Effect of shape and size of sampling window on the determination of average length, intensity and density of trace discontinuity," in Proceedings of Rock Mechanics and Rock Engineering: From the Past to the Future, pp. 1073-1078, Urgup, Turkey, August 2016.

[23] S. Mahé, M. Gasc-Barbier, and R. Soliva, "Joint set intensity estimation: comparison between investigation modes," Bulletin of Engineering Geology and the Environment, vol. 74, no. 1, pp. 171-180, 2015.

[24] H. Watkins, C. E. Bond, D. Healy, and R. W. H. Butler, "Appraisal of fracture sampling methods and a new workflow to characterise heterogeneous fracture networks at 
outcropflow to characterise heterogeneous fracture networks at outcrop," Journal of Structural Geology, vol. 72, pp. 67-82, 2015.

[25] O. J. Ortega, R. A. Marrett, and S. E. Laubach, "A scale-independent approach to fracture intensity and average spacing measurement," AAPG Bulletin, vol. 90, no. 2, pp. 193-208, 2006.

[26] P. H. S. W. Kulatilake and T. H. Wu, "The density of discontinuity traces in sampling windows," International Journal of Rock Mechanics and Mining Sciences \& Geomechanics Abstracts, vol. 21, no. 6, pp. 345-347, 1984.

[27] Q. Wu, P. H. S. W. Kulatilake, and H.-m. Tang, "Comparison of rock discontinuity mean trace length and density estimation methods using discontinuity data from an outcrop in Wenchuan area, China," Computers and Geotechnics, vol. 38, no. 2, pp. 258-268, 2011.

[28] P. H. S. W. Kulatilake and T. H. Wu, "Estimation of mean trace length of discontinuities," Rock Mechanics and Rock Engineering, vol. 17, no. 4, pp. 215-232, 1984.

[29] D. N. Wathugala, P. H. S. W. Kulatilake, G. W. Wathugala, and O. Stephansson, "A general procedure to correct sampling bias on joint orientation using a vector approach," Computers and Geotechnics, vol. 10, no. 1, pp. 1-31, 1990. 